\title{
Age Estimates for Galaxies in Groups
}

\author{
Duncan A. Forbes and Alejandro I. Terlevich \\ School of Physics and Astronomy, University of Birmingham, \\ Birmingham B15 2TT, UK
}

\begin{abstract}
.
We discuss recent developments on the age and metallicity distribution for early type galaxies in different environments.
\end{abstract}

\section{Introduction}

A galaxy's environment plays a key role in determining its evolution. For elliptical galaxies, it is generally thought that mergers of disk galaxies in the field and in groups are the dominant formation mechanism. Elliptical-rich groups that fall in along filaments create the elliptical-rich clusters we see today. Tracking the assembly of elliptical galaxies and the evolutionary status of groups would provide further insight into these processes.

Until recently it was very difficult to directly age-date the stars in old stellar populations due to the age-metallicity degeneracy. This degeneracy has now been broken by new spectroscopic observations and models (e.g. Worthey 1994; Trager et al. 1999). Thus it is now possible to form an evolutionary sequence of elliptical galaxy formation and to age-date the ellipticals in different environments.

\section{Deviations from Galaxy Scaling Relations}

In two recent papers (Forbes et al. 1998; Forbes \& Ponman 1999) we showed that a galaxy's position relative to the fundamental plane and other scaling relations depends on a galaxy's age. Here age is the central luminosity weighted age of the galaxy from stellar spectroscopy. We found that young ellipticals were brighter with a higher surface brightness. Ellipticals that were $\sim 10 \mathrm{Gyr}$ old would lie on the FP. From simple starburst models, we showed that fading central starbursts could explain the overall trend. The situation was similar for the deviations from $2 \mathrm{D}$ scaling relations such as $B-V$ vs $\mathrm{M}_{B}$ and $\mathrm{Mg}_{2}-\sigma$. Younger galaxies would redden and their $\mathrm{Mg}_{2}$ line strengths weaken as the central starburst faded. We concluded that these scaling relations are metallicity-mass sequences with deviations caused by a galaxy's age. 


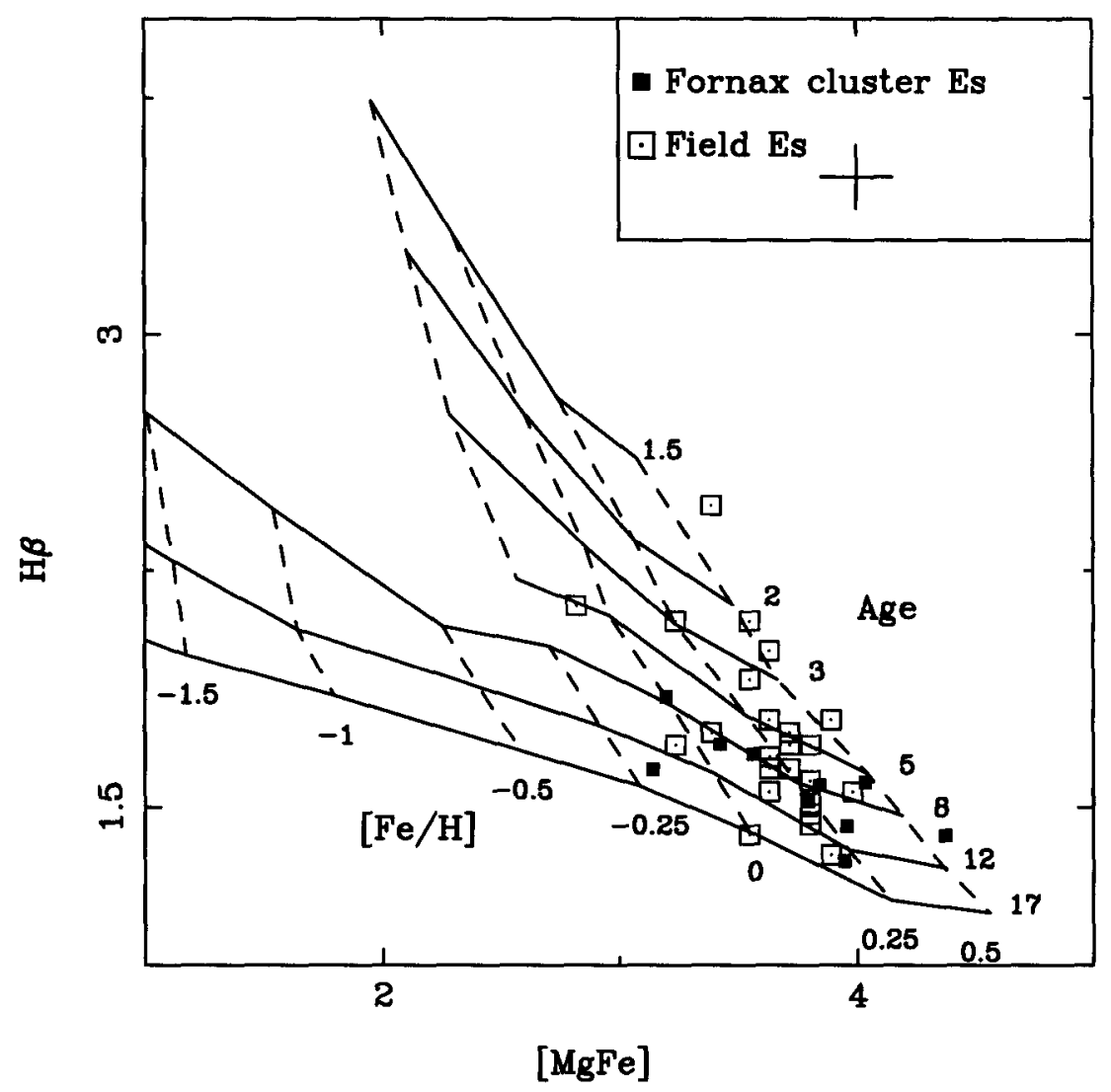

Figure 1. Field and Fornax cluster ellipticals from Gonzalez (1993) and Kuntschner \& Davies (1998). The cluster ellipticals are all about 8 Gyrs old, whereas the field ellipticals appear to scatter in an age sequence at constant metallicity. 


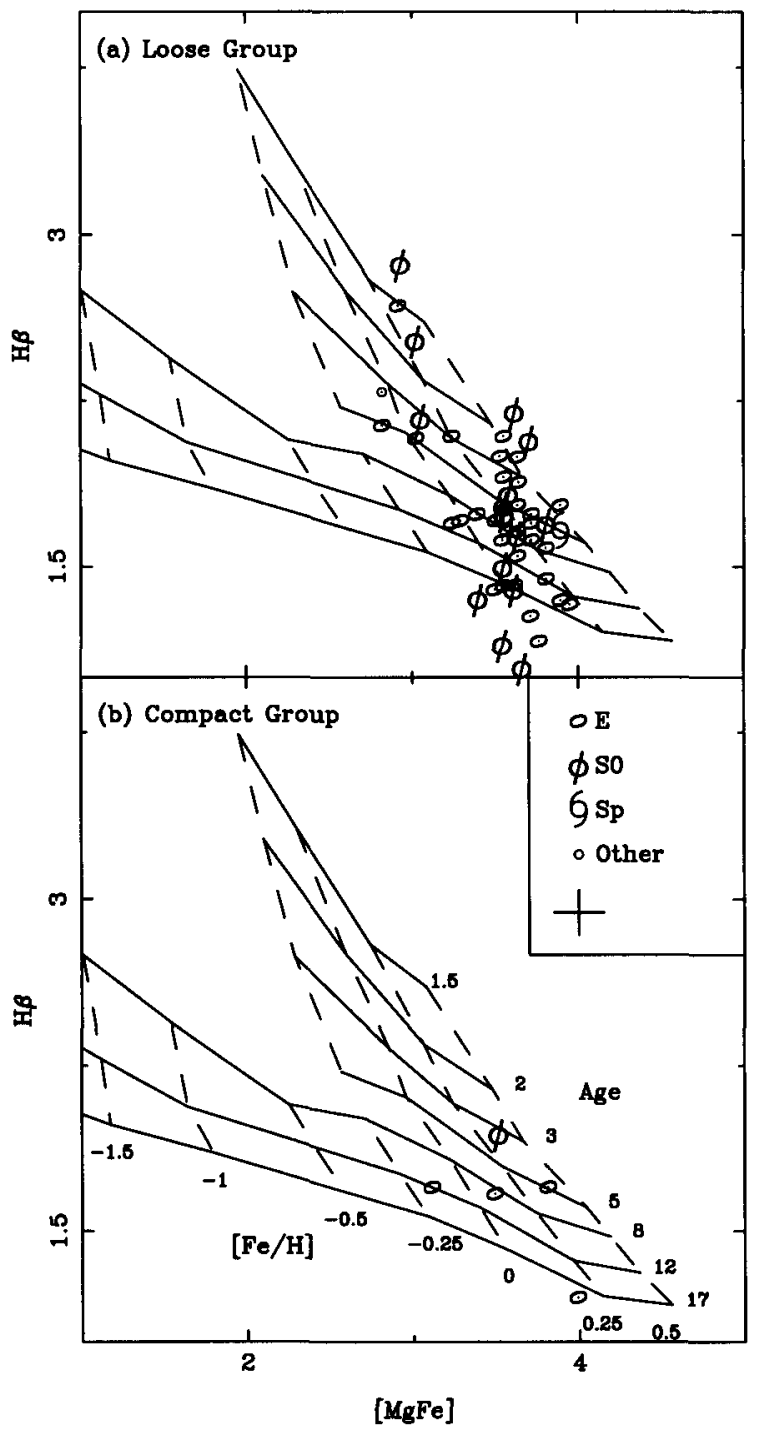

Figure 2. Loose and compact group early type galaxies from the literature. Most loose group ellipticals appear to be old. There are too few compact group ellipticals studied to date to describe their age and/or metallicity distibution. 


\section{The Age and Metallicity Distribution of Galaxies}

Perhaps the best, high quality study of field ellipticals is that of Gonzalez (1993). He obtained new absorption line indices for about 40 early type galaxies in the field, and claimed that when plotted on a Worthey (1994) grid of $\mathrm{H} \beta$ vs $[\mathrm{MgFe}]$ they generally scatter across a range in ages with metallicities concentrated around solar. Another high quality study is that of Kuntschner \& Davies (1998) who studied early type galaxies in the Fornax cluster. They found all ellipticals to have a similar age of $\sim 8$ Gyrs, covering a range in metallicity. Only the So galaxies scattered to young ages in the Worthey grid. There is certainly support from the Coma cluster that the colour-magnitude relation is largely a metallicity-mass sequence with the small scatter due to age effects (Terlevich et al. 1999). These field and cluster samples are shown in Fig. 1. Although the cluster galaxy trends are fairly convincing, more field data is needed to confirm the Gonzalez claims.

If field ellipticals appear to describe a sequence in age, while cluster ellipticals describe a sequence in metallicity (at constant age), how do group ellipticals behave?

- If group ellipticals resemble cluster ellipticals, then it suggests that 'evolutionary' processes have already occured, and must be related to non-cluster environments, e.g. merging.

- If group ellipticals resemble field ellipticals, then it suggests that 'evolutionary' processes have yet to occur, and must be related to cluster environments, e.g. ram pressure stripping, harassment.

An $\mathrm{H} \beta$ vs $[\mathrm{MgFe}]$ plot for loose groups and compact groups is shown in Fig. 2. In both cases, most early type galaxies are old ( $\sim 10 \mathrm{Gyr})$, with some of young age but there are too few to make conclusive statements. However building up large samples of group galaxies with age estimates should provide unique clues to their star formation histories, and in the case of compact groups - the evolutionary status of the group itself.

Acknowledgments. We would like to thank R. Brown and T. Ponman for their contributions to this work. The IAU kindly provided travel assistance to the meeting.

\section{References}

Forbes, D., Ponman, T., Brown, R., 1998, ApJ, 508, L43

Forbes, D., Ponman, T., 1999, MNRAS, 309, 623

Gonzalez, J. 1993, PhD Thesis, Santa Cruz

Kuntschner, H., Davies, R. L. 1998, MNRAS, 295, 29

Terlevich, A., et al. 1999, MNRAS, in press

Trager, S., et al. 1999, in preparation

Worthey, G., 1994, ApJS, 95, 107 